\title{
The Glucagon-Like Peptide-1 Analogue Liraglutide Inhibits Oxidative Stress and Inflammatory Response in the Liver of Rats with Diet-Induced Non-alcoholic Fatty Liver Disease
}

\author{
Huiting Gao, Zhigang Zeng, Han Zhang, Xiaoli Zhou, Lichang Guan, Weiping Deng, and \\ Lishu $\mathrm{Xu}^{*}$ \\ Department of Gastroenterology, Guangdong General Hospital, Guangdong Academy of Medical Sciences, Institute \\ of Geriatrics; Guangzhou 510080, China. \\ Received July 10, 2014; accepted February 16, 2015
}

\begin{abstract}
Liraglutide, a glucagon-like peptide-1 (GLP-1) analogue, has been demonstrated to reduce hepatic steatosis. However, the mechanism of the lipid-lowering effect of liraglutide in the liver remains unclear. The aim of the present study was to investigate the beneficial effect of liraglutide on diet-induced non-alcoholic fatty liver disease (NAFLD) and the underlying mechanism in rats. NAFLD was induced in Sprague-Dawley rats by feeding a high fat and high cholesterol (HFHC) diet. Liraglutide $(0.6 \mathrm{mg} / \mathrm{kg}$ body weight/d) was injected intraperitoneally to the rats subjected to HFHC diet four weeks before sacrificing the animals. Body and liver weight, fasting blood glucose (FBG), fasting insulin, serum aminotransferase (ALT) and lipid accumulation in the liver were determined. Markers of oxidative stress, such as malondialdehyde (MDA), free fatty acid (FFAs), superoxide dismutase (SOD), and pro-inflammatory cytokine tumor necrosis factor- $\alpha$ (TNF- $\alpha$ ) were detected by colorimetric detection or enzyme-linked immunosorbent assay (ELISA). Serum and hepatic adiponectin were measured by ELISA. The expression of c-Jun N-terminal kinase-1 (JNK-1) and phosphorylated JNK-1 were examined by Western blotting. Liraglutide improved insulin resistance, decreased hepatic steatosis and reversed liver dysfunction. The hepatic levels of MDA, FFAs, and TNF- $\alpha$ were significantly decreased versus controls. Meanwhile, administration of liraglutide significantly increased SOD and adiponectin levels in the liver and inhibited the expression of JNK-1 and phosphorylated JNK-1 versus control rats. Liraglutide exerted anti-oxidative and anti-inflammatory effects in the liver and consequently reversed hepatic steatosis and insulin resistance. Such effects might be mediated by the elevation of adiponectin levels and the inactivation of JNK-1.
\end{abstract}

Key words liraglutide; non-alcoholic fatty liver disease; oxidative stress; inflammatory response; adiponectin

Non-alcoholic fatty liver disease (NAFLD), one of the most common liver diseases in the world, is closely associated with insulin resistance and is considered the hepatic manifestation of the metabolic syndrome. ${ }^{1-3)}$ NAFLD represents a spectrum of disease ranging from simple steatosis to steatohepatitis (NASH), increasing fibrosis and, eventually, cirrhosis and hepatocellular carcinoma. ${ }^{4,5)} \mathrm{Up}$ to 10 to $25 \%$ patients with NAFLD progress to NASH and 10 to $15 \%$ patients with NASH develop hepatocellular carcinoma., ${ }^{6,7)}$ Although the pathogenesis of NAFLD remains elusive, the prevailing theory for the development of NAFLD is the 'two-hit' hypothesis. The first hit is the dysregulation of fatty acid metabolism, which leads to simple steatosis. The first hit renders hepatocytes susceptible to the secondary hit, including oxidative stress and inflammatory response, which further results in NASH or even cirrhosis. ${ }^{8-10)}$ At present, there are no standard therapeutic strategies for NAFLD management. The current treatment for NAFLD is limited to improving insulin sensitivity with lifestyle interventions and weight loss, sometimes through bariatricsurgery. ${ }^{11)}$ Since lifestyle modifications and weight loss remain difficult to achieve by most people, an effective pharmacologic treatment for NAFLD is sorely needed. ${ }^{12,13)}$

Liraglutide, a glucagon-like peptide-1 (GLP-1) analogue with $97 \%$ sequence identity to human GLP-1, is known to decrease blood glucose, through potentiating glucose-dependent insulin secretion, enhancing beta-cell growth, and reducing food intake and body weight. ${ }^{14,15)}$ Studies in the liver and adipose tissue of diabetic mice have demonstrated that GLP-1 improves insulin resistance and reduces hepatic steatosis. ${ }^{16-18)}$ It has been reported that liraglutide reduces tumor necrosis factor (TNF)- $\alpha$ or high glucose induced oxidative stress and inflammatory response in human endothelial cells. ${ }^{19,20)}$ Inhibition of protein kinase $\mathrm{C}$ (PKC) translocation, reduced nicotinamide adenine dinucleotide phosphate (NADPH) oxidase activation and nuclear factor (NF)- $\kappa \mathrm{B}$ activation may contribute to the anti-oxidative and anti-inflammatory effects of liraglutide. ${ }^{19,20)}$ Recently, liraglutide has been reported to reduce the level of plasma reactive oxygen metabolites, an indicator of oxidative stress, in patients with type 2 diabetes. ${ }^{21)}$ In addition, the anti-inflammatory effect of liraglutide has been demonstrated in the heart of mice with diabetes and the brain of mice with Alzheimer's disease. ${ }^{22,23)}$ We have previously published preliminary data of the hepatic effect of GLP-1 on oxidative stress and inflammatory factors. ${ }^{24)}$ The present study aimed to explore the underlying mechanisms.

However, whether liraglutide can reverse oxidative stress and inflammation in the liver still remains obscure. Adiponectin, a specific protein secreted by adipocytes, has been demonstrated to play an important role in the pathogenesis of NAFLD due to its insulin-sensitizing, antifibrogenic and anti-inflammatory properties by action on hepatocytes, hepatic stellate cells and Kupffer cells, respectively. ${ }^{25)}$ Polyzos et al. have reported that adiponectin inhibits the expression, 


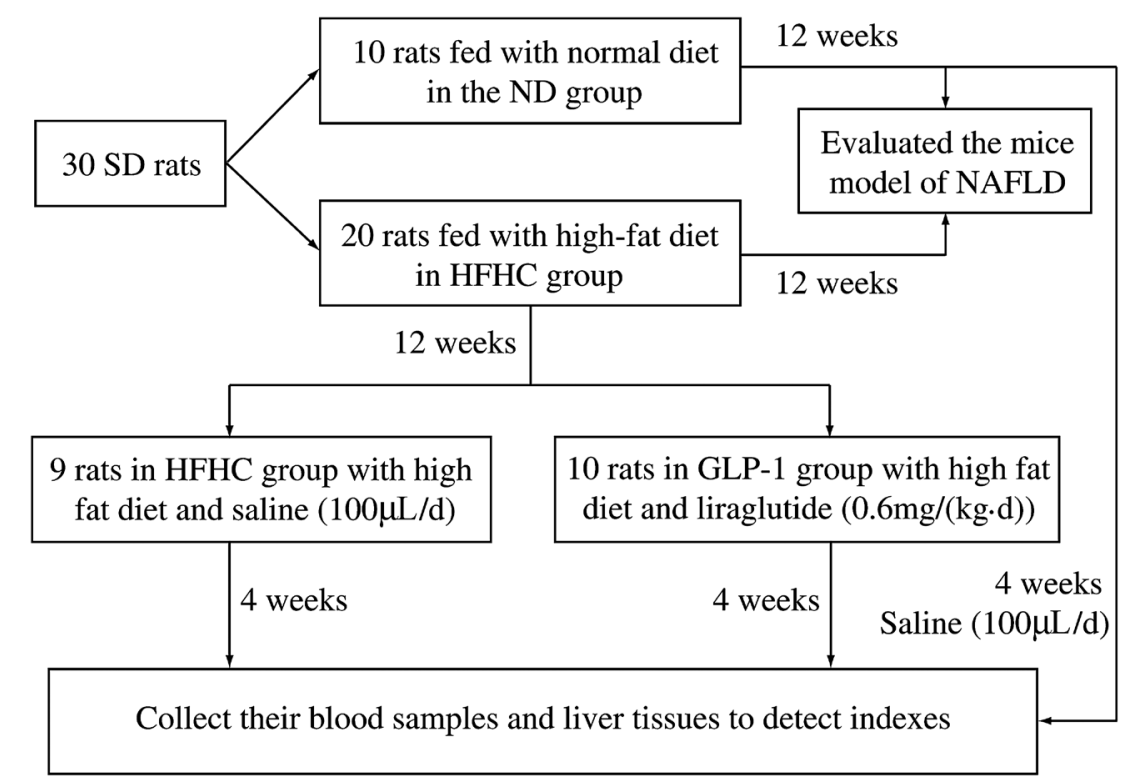

Fig. 1. Schematic Diagram of the Experimental Design

From the 12 th week until the 16 th week, the rats were injected with saline or liraglutide. At the end of the 16th week, the liver tissues and blood samples were harvested for evaluation.

secretion and action of TNF- $\alpha$, thereby improving insulin sensitivity, whereas TNF- $\alpha$ suppresses adiponectin transcription, secretion and action, thereby aggravating insulin resistance. ${ }^{26)}$ Additionally, a significant correlation between adiponectin, inflammatory markers and oxidative stress has been observed in patients with metabolic syndrome. ${ }^{27)}$ Liraglutide has been reported to significantly increase plasma adiponectin levels and adiponectin protein expression in adipose tissue in the mice with high fat diet-induced NAFLD. ${ }^{28)}$ However, whether liraglutide can also elevate the adiponectin levels in the liver is not yet established. In addition, the c-Jun N-terminal kinase (JNK) signal transduction pathway as a link between inflammation and metabolic diseases plays a pivotal role in the development of NAFLD and insulin resistance. ${ }^{29,30)}$ The activation of JNKs (mainly JNK1) is found to mediate insulin resistance, steatohepatitis and inflammatory response. ${ }^{31,32)} \mathrm{Ad}-$ iponectin has been demonstrated to be elevated in the serum of obese $\mathrm{JNK}^{-1-}$ mice. ${ }^{33)}$ Whether JNK mediates the beneficial effect of liraglutide in the liver via adiponectin warrants more comprehensive investigation.

We hypothesized that liraglutide improves liver function and insulin resistance via inhibiting oxidative stress and inflammatory response in the liver during NAFLD. In the present study, we investigated the effects and mechanisms of liraglutide on high fat and high cholesterol diet-induced NAFLD in rats. We found that treatment with liraglutide for four weeks significantly inhibited oxidative stress and inflammatory markers in the rat liver and that the underlying mechanisms may be associated with the elevation of adiponectin levels and the inactivation of the JNK signaling pathway.

\section{MATERIALS AND METHODS}

Animals Thirty 5-week-old male Sprague Dawley (SD) rats (Experimental Animal Center of Zhongshan Medicine University, Guangzhou, China) were fed for 16 weeks with (1) regular rodent diet (ND group) containing 4.3\% lard (w/w) and no cholesterol; (2) high fat and high cholesterol diet (HFHC group) containing $88 \%$ ordinary forage, $10 \%$ lard and $2 \%$ cholesterol (w/w); (3) high fat and high cholesterol diet and daily intraperitoneal injection of liraglutide $(0.6 \mathrm{mg} / \mathrm{kg}$ body weight, intraperitoneally (i.p.)) for four weeks before euthanasia (HFHC-L group). The rats in the ND and HFHC groups were given saline intraperitoneally daily for four weeks before euthanasia instead of liraglutide. Each group contained ten rats. A schematic diagram of the animal experiment design is shown in Fig. 1. All rats had free access to food and water and were kept on a 12 -h light $/ 12$-h dark cycle $\left(20 \pm 2^{\circ} \mathrm{C}\right.$, lights on 7:00 a.m. to 19:00 p.m.). At the end of the 16 th week, all rats were sacrificed after $12 \mathrm{~h}$ of fasting. Serum and liver tissue were collected for further examination. The liver index was calculated as liver weight/body weight $\times 100 \%$. All procedures and treatment of mice were reviewed and approved by the ethical committee at Guangdong General Hospital (Guangdong Academic of Medical Sciences, Guangzhou, China).

Blood Chemistry Blood from postcava vein was collected for further measurement of fasting blood glucose (FBG), aminotransferase (ALT), triglyceride (TG), total-cholesterol (TC), fasting insulin (FINS) and adiponectin. Serum FBG, ALT and lipids were detected with a biochemistry automatic analyzer (Beckman Coulter, U.S.A.). FINS and adiponectin were measured by ELISA (Shanghai ExCell Biology, Inc.). Homeostasis assessment-insulin resistance (HOMA-IR) was expressed as [FBG $\times$ FINS] $/ 22.5$ according to the manufacturer's instructions.

Hepatic Parameters Lipids in the liver were extracted using a previously described protocol. ${ }^{34)}$ In brief, $0.2 \mathrm{~g}$ frozen liver tissue was rinsed of blood and placed in $1.8 \mathrm{~mL}$ of ice-cold homogenization buffer (chloroform-methanol, $2: 1 \mathrm{v} / \mathrm{v}$ ) and then sliced into sections on ice. The tissue was homogenized on ice for 3-5 min. The homogenate was then centrifuged at $2000 \mathrm{rpm}$ for $15 \mathrm{~min}$. The clarified bottom layer was then apportioned into $1 \mathrm{~mL}$ aliquots and stored at $-70^{\circ} \mathrm{C}$ until ready for use. Intrahepatic lipids were quantified spectro- 
photometrically using an enzymatic assay (Nanjing Jiancheng Bioengineering Institute) and expressed as lipid (mmol)/g wet liver weight.

Hepatic malondialdehyde (MDA) levels were determined using the thiobarbituric acid reactive substances assay (TBA), which measures malondialdehyde equivalent 35). The methods for measuring the superoxide dismutase (SOD) and free fatty acid (FFAs) have been previously described. ${ }^{36,37)}$ The levels of SOD, MDA and FFAs in liver homogenates were detected by colorimetric detection (Nanjing Jiancheng Bioengineering Institute).

The procedures for determining rat TNF- $\alpha$ and adiponectin concentrations in homogenates of rat livers have been previously described, ${ }^{38,39)}$ and were detected by ELISA (Nanjing Jiancheng Bioengineering Institute).

Histological Staining A portion of liver was fixed in $3.6 \%$ formaldehyde (formalin) overnight and then embedded in paraffin. The paraffin-embedded liver tissues were sectioned $(5 \mu \mathrm{m})$ and stained with hematoxylin and eosin (H\&E) to evaluate the morphological changes in the liver. The images of H\&E sections (5 per liver) were captured by light microscopy (Leica DM2500, Germany).

Another portion of liver was frozen immediately in liquid nitrogen after the liver was excised. Cryosections of the liver tissue were prepared and stained with Oil Red $\mathrm{O}$ to visualize lipid droplets as previous described. ${ }^{40)}$ In brief, sections were stained in $0.7 \%$ Oil Red $\mathrm{O}$ solution for $10-15 \mathrm{~min}$ followed by counterstaining with Harris Hematoxylin for $1 \mathrm{~min}$ after washing. Sections were captured and analyzed by light microscopy (Leica DM2500).

Western Blot Analysis Approximately $0.1 \mathrm{~g}$ liver tissue was crushed in liquid nitrogen, and then homogenized in $1 \mathrm{~mL}$ of ice-cold lysis buffer with protease inhibitors and phosphatase inhibitors (Roche). The homogenate was then centrifuged at $12000 \times \boldsymbol{g}$ for $30 \mathrm{~min}$ and the supernatant was harvested. The protein concentration of the supernatant was measured with a bicinchoninic acid (BCA) protein quantification kit (Beyotime Institute of Biotechnology). Liver proteins $(60 \mu \mathrm{g})$ were separated by $10 \%$ sodium dodecyle sulfate (SDS)-polyacrylamide gel electrophoresis and then transferred to polyvinylidene (PVDF) membranes (Millipore). Membranes were blocked in Tris-buffered saline (TBS) containing $0.1 \%$ Tween-20 and 5\% skim milk for $2 \mathrm{~h}$ at room temperature. Afterwards, membranes were incubated with primary antibody, including anti-JNK1 [Abcam; 1:2000 dilution with bovine serum albumin (BSA) blocking buffer $(5 \%$ (w/v) BSA in phosphate buffered saline)], anti-phospho-JNK1 (Abcam; 1:1000 dilution with the $5 \%(\mathrm{w} / \mathrm{v})$ BSA blocking buffer) or $\beta$-actin (Abcam; 1:1000 dilution with the 5\% (w/v) BSA blocking buffer) overnight at $4^{\circ} \mathrm{C}$. Following three consecutive $5 \mathrm{~min}$ washes in TBS with Tween-20 (TBST) and incubation with horseradish peroxidase (HRP)-conjugated secondary antibody (Invitrogen; 1:5000 dilution with the 5\% (w/v) BSA blocking buffer) for $2 \mathrm{~h}$ at $4^{\circ} \mathrm{C}$, protein expression was detected with enhanced chemiluminescence and densitometry was performed using a FluorChem 8900 System software.

Statistical Analyses Data were analyzed and curves fitted with SPSS 16.0 software. Data were presented as mean \pm S.D. Multiple comparisons were analyzed by one way ANOVA followed by post hoc testing using least significance difference (LSD) for multiple comparisons. A value of $p<0.05$ was considered statistically significant.

\section{RESULTS}

Liraglutide Decreases Body Weight and Liver Index, and Improves Liver Function in HFHC Rats Administration of liraglutide significantly reduced the body weight (by $11 \%)$ and liver index as compared to HFHC group $(p<0.05)$ (Figs. 2A, B). As shown in Fig. 2C, serum ALT levels of the
A
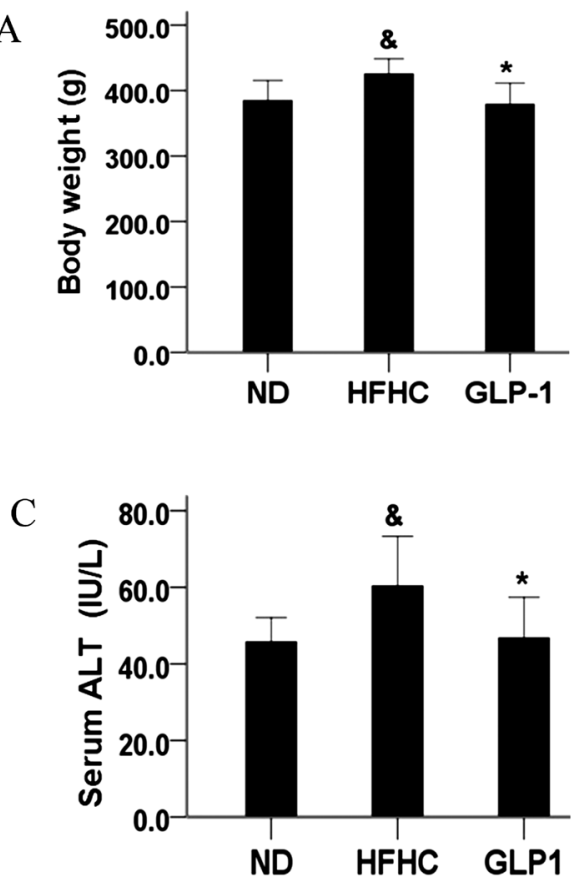

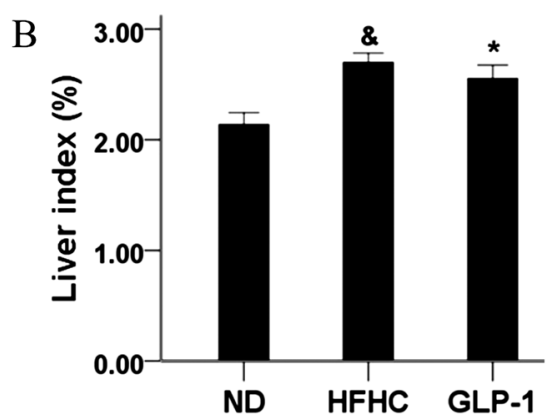

Fig. 2. The Effect of Liraglutide on Body Weight, Liver Index and Liver Function

(A) Body weight of the rats; (B) liver index of the rats; (C) serum ALT levels; ${ }^{\&} p<0.05$ compared to the ND group. ${ }^{*} p<0.05$ compared to the HFHC group. 


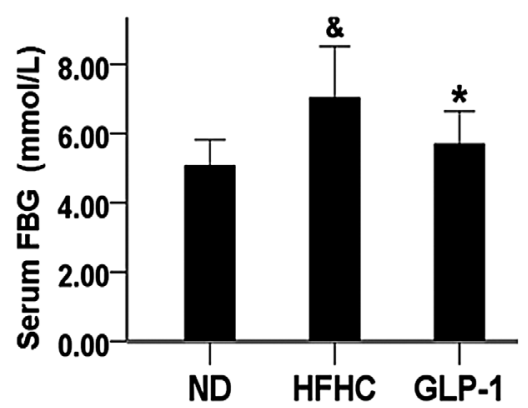

A

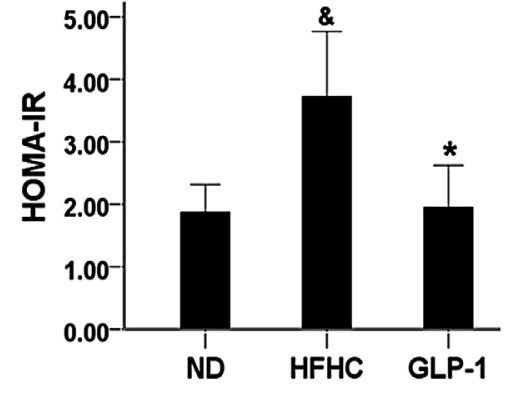

B

Fig. 3. The Effects of Liraglutide on Glucose Metabolism and Homeostasis Assessment-Insulin Resistance (HOMA-IR)

(A) Serum levels of FBG; (B) levels of HOMA-IR; ${ }^{\star} p<0.05$ compared to the ND group. ${ }^{*} p<0.05$ compared to the HFHC group.
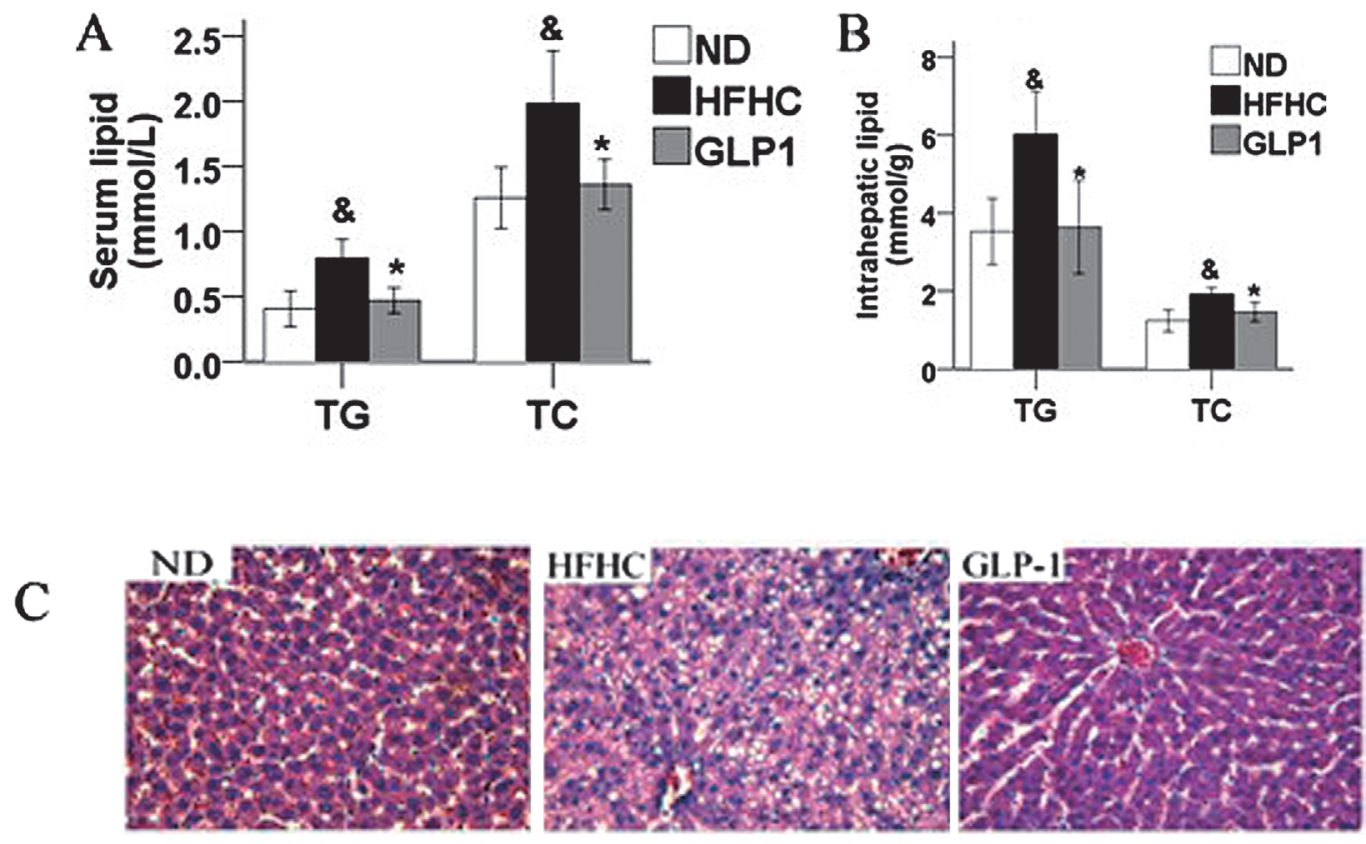

D
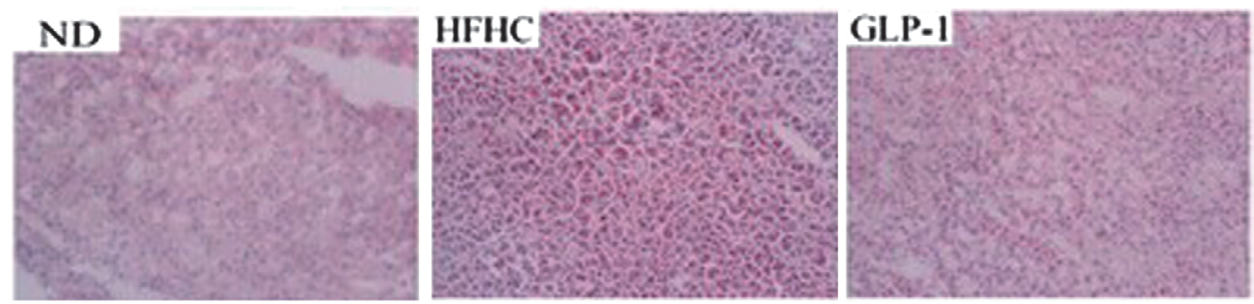

Fig. 4. Liraglutide Reduces Lipid Accumulation and Improves Pathological Indexes in the Liver

(A) Serum levels of TC and TG; (B) levels of TC and TG in liver homogenates; (C) histology of rat livers (HE $\times 400)$; (D) histology of the rat livers (Oil red O $\times 200$ ). ${ }_{p}<0.05$ compared to the ND group. ${ }^{*} p<0.05$ compared to the HFHC group.

liraglutide-treated rats with liraglutide were markedly decreased compared to the HFCH group $(49 \pm 11$ vs. $59 \pm 12 \mathrm{IU} / \mathrm{L}$, respectively, $p<0.05$ ).

Liraglutide Improves Glucose Metabolism and Homeostasis Assessment-Insulin Resistance (HOMA-IR) As shown in Fig. 3, FBG and HOMA-IR levels in the rats fed a high fat and high cholesterol diet were singificantly higher than those in their control counterparts fed with regular diet $(p<0.05)$. According to the American Diabetes Association, HOMA-IR $>2.69$ is considered as the standard for insulin resistance. Compared with the rats in the ND and HFHC-L groups, rats in the HFHC group showed more abnormalities in glycemic metabolism, including insulin resistance $(p<0.05)$. After treatment with liraglutide for 4 weeks, the levels of FBG and HOMA-IR in the HFHC rats were reduced to the ND group levels (Figs. 3A, B).

Liraglutide Reduces Lipid Accumulation in the Serum and Liver Compared to the ND group, serum levels of TC and TG in the HFHC rats were singificantly higher $(1.98 \pm 0.40$ vs. $1.26 \pm 0.24 \mathrm{mmol} / \mathrm{L}$, respectively; $0.80 \pm 0.14$ vs. $0.41 \pm 0.13 \mathrm{mmol} / \mathrm{L}$, respectively; Fig. $4 \mathrm{~A}$ ). In addition, liraglutide treatment significantly reduced the elevation of 
A

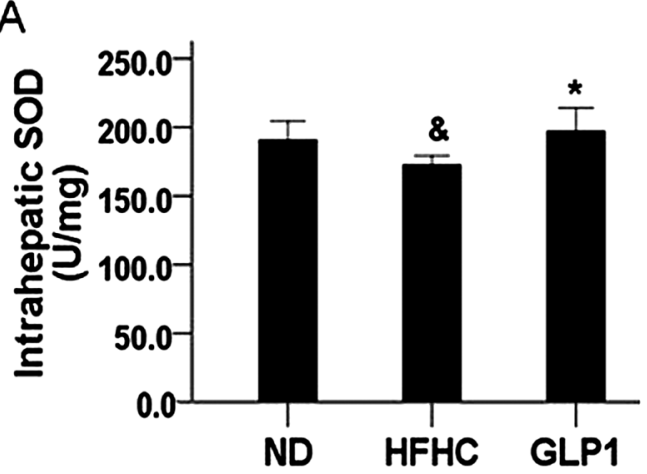

C

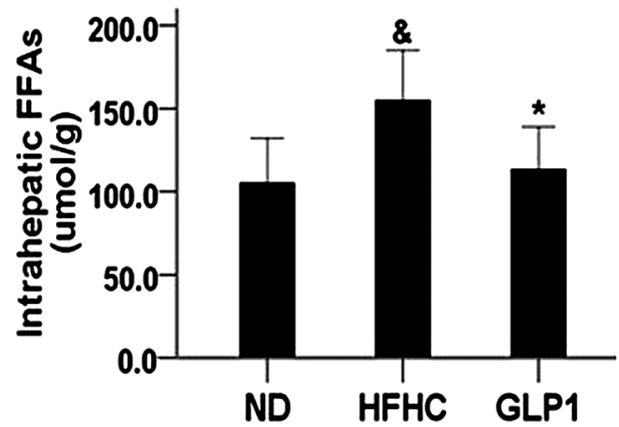

B

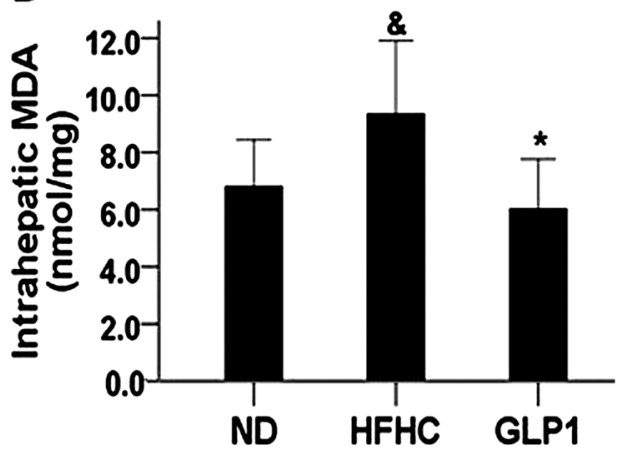

D

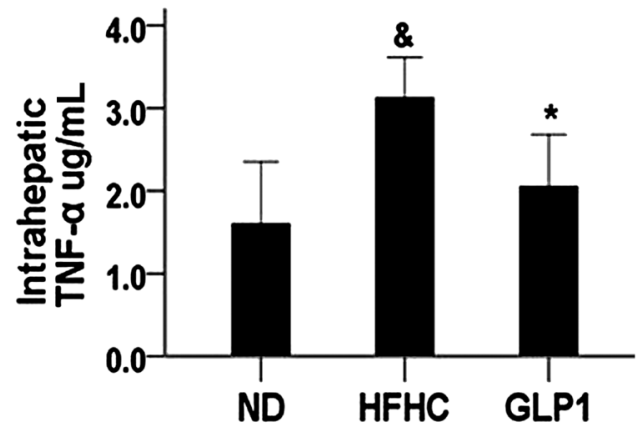

Fig. 5. Liraglutide Improves Oxidative Stress and Lipid Peroxidation and Decreases Inflammation

(A) Levels of SOD in liver homogenates; (B) levels of MAD in liver homogenates; (C) levels of FFAs in liver homogenates; (D) levels of TNF- $\alpha$ in liver homogenates; $\& p<0.05$ compared to the ND group. ${ }^{*} p<0.05$ compared tothe HFHC group.

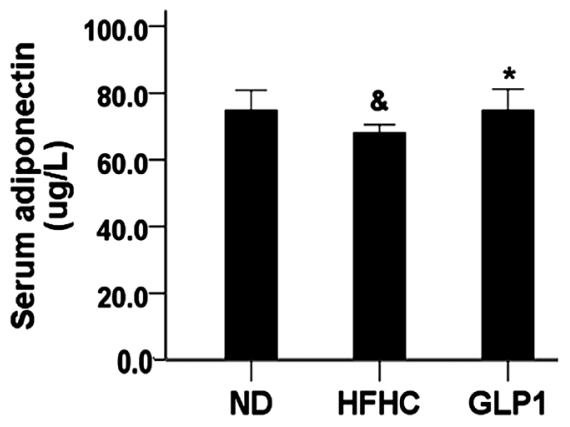

A

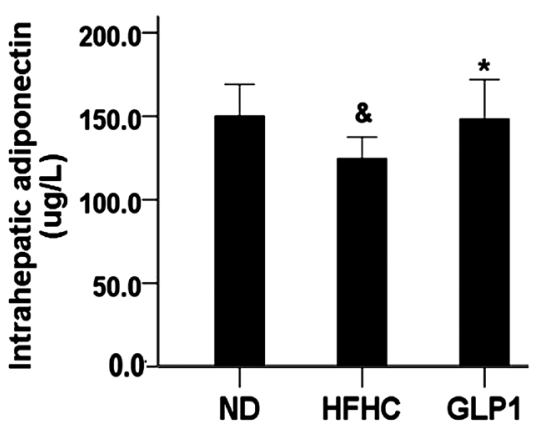

B

Fig. 6. The Effect of Liraglutide on Serum Adiponectin Levels

(A) Serum levels of adiponectin; (B) levels of ADP liver homogenates; ${ }^{\&} p<0.05$ compared to the ND group. ${ }^{*} p<0.05$ compared to the HFHC group.

TC and TG compared to the HFHC group $(1.36 \pm 0.20$ vs. $1.98 \pm 0.40 \mathrm{mmol} / \mathrm{L}$, respectively; $0.47 \pm 0.10 \mathrm{vs}$. $0.80 \pm 0.14 \mathrm{mmol} / \mathrm{L}$, respectively; Fig. 4A). In accordance, administration of liraglutide also significantly inhibited the TC and TG levels in the liver (Fig. 4B). Such changes of hepatic lipid content were confirmed by $\mathrm{H} \& \mathrm{E}$ and Oil Red $\mathrm{O}$ staining (Figs. 4C, D). Hepatic steatosis and ballooning degeneration of hepatocytes were visualized by H\&E staining. Oil Red O staining revealed numerouslipid droplets in the rat liver of the HFHC group. However, no evidence of hepatic steatosis was observed in the rats fed a regular diet (ND group) or treated with liraglutide.

Liragltuide Exerts Anti-oxidative and Anti-inflammatory Effects in the Liver The level of MDA, a stable end product of lipid peroxidation that served as an indicator of oxidative stress in the liver, was measured. MDA levels were significantly elevated in the liver of HFHC rats, while liraglutide reduced the hepatic MDA levels versus the HFHC group (Fig. 5B). It is well known that excess FFA can proceed with lipid peroxidation and then produce a large number of reactive oxygen species (ROS), which causes oxidative stress. ${ }^{41}$ ) Our results showed that the levels of FFAs in the liver were significantly decreased by liraglutide treatment (Fig. 5C). To further investigate the effect of liraglutide on the intracellular superoxide levels, the SOD levels were measured in the rat liver. It was found that the level of SOD was significantly elevated by the administration of liraglutide compared to HFHC group (193.4 \pm 7.1 vs. 172.3 $\pm 6.3 \mathrm{U} / \mathrm{mg}$; Fig. 5A). In addition, the levels of TNF- $\alpha$, a pro-inflammatory marker, were also significantly reduced after liraglutide treatment (Fig. 5D). These results indicate that liraglutide inhibits oxidative stress 
ND HFHC GLP1

A

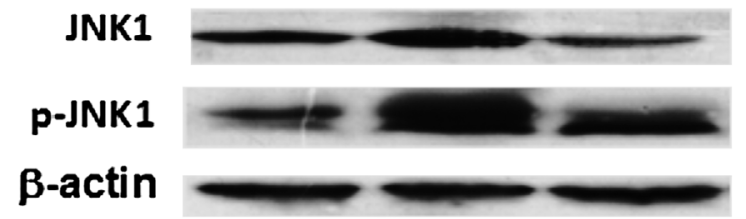

$46 \mathrm{kDa}$

$48 \mathrm{kDa}$

42 kDa
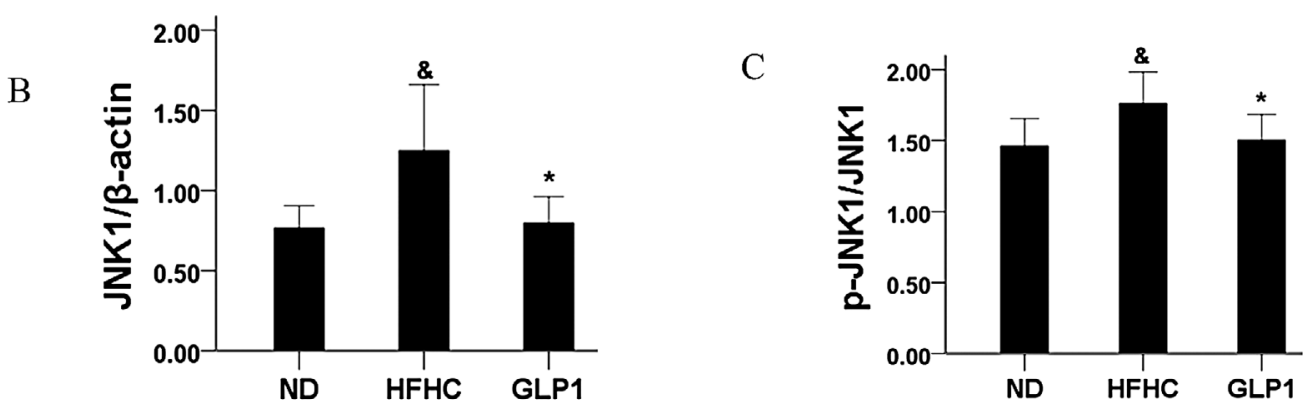

Fig. 7. Expression Levels of JNK1 and p-JNK1 in Liver Homogenates Detected by Western Blotting

(A) Expression of JNK1; (B) p-JNK1 expression level normalized to JNK1; ${ }^{\&} p<0.05$ compared to the ND group. ${ }^{*} p<0.05$ compared to the HFHC group.

via the reduction of lipid peroxidation and activation of SOD, and suppresses the inflammatory response in the liver.

Liraglutide Elevates Serum and Liver Adiponectin Levels Compared to the ND group, the levels of adiponectin in the serum and liver homogenates in HFHC rats were significantly decreased. Liraglutide significantly increased these levels (Figs. 6A, B).

The Effect of Liraglutide Involves the JNK Pathway Our results showed that the protein expression of JNK1, phosphorylated JNK1 (p-JNK1) and the ratio of p-JNK1 to JNK1 were significantly increased in the HFHC group $(p<0.05)$. Such changes were completely reversed by 4 -week treatment with liraglutide $(p<0.05)$ (Fig. 7).

\section{DISCUSSION}

Oxidative stress and inflammatory response have been demonstrated to be the second hit for the development of NAFLD. ${ }^{8)}$ Liraglutide, a glucagon-like peptide-1 analogue, has been found to inhibit oxidative stress and inflammatory response in the endothelial cells, heart and brain. ${ }^{19,20,22,23)} \mathrm{Re}$ sults obtained from the present study contribute to further understanding of the mechanisms whereby liraglutide exerts a protective effect against high fat and high cholesterol diet induced hepatic steatosis, insulin resistance and liver dysfunction. Administration of liraglutide effectively alleviated hepatic oxidative stress and inflammatory response. Such antioxidative and anti-inflammatory effects of liraglutide might be mediated by the inactivation of JNK pathway and the elevation of adiponectin in the liver.

NAFLD is a clinicopathological syndrome including a wide spectrum of liver damage, characterized by steatosis along with inflammation and hepatocellular damage in patients with no history of significant alcohol consumption. ${ }^{42)}$ The pathogenesis of NAFLD has not been fully clarified. In the present study, high fat and high cholesterol diet was employed to induce NAFLD in the rats. Our results demonstrated that hepatic steatosis and insulin resistance were successfully in- duced in the rats by feeding the animals a high fat and high cholesterol diet for 16 weeks. In addition to the first hit of lipid accumulation, oxidative stress and inflammation are regarded as the second hit in the pathogenesis of NAFLD in the "two-hit" theory. ${ }^{8)}$ When NAFLD occurs, excess FFAs in the liver cells lead to the development of lipid peroxidation and the generation of a large number of ROS, which are the major indicators of oxidative stress. ${ }^{43)}$ Consequently, ROS prompt the generation of TNF- $\alpha$, which inhibits mitochondria respiration and induces and aggravates insulin resistance. ${ }^{44,45)}$ Superoxide dismutase (SOD) is an important antioxidant enzyme and the primary means of free radicals scavenging in organisms. ${ }^{46}$ ) Malondialdehyde (MDA) is the advanced lipoxidation end product of the peroxidation reaction, which results from the action of free radicals on lipids. ${ }^{41)}$ The amount of hepatic MDA can indirectly reflect the degree of cell damage. The combination of SOD and MDA is usually used to evaluate the oxidative stress level of cells. ${ }^{47)}$ Our results demonstrated that the levels of MDA, FFAs, and TNF- $\alpha$ were remarkably increased while the SOD levels were significantly decreased in the liver of rats fed with high fat and high cholesterol diet as compared to ND rats. These results were in accordance with previous reports of rodents fed with a high fat or high cholesterol diet. ${ }^{48)}$

GLP-1, a glucose-dependent incretin, exerts its beneficial effects by increasing fatty acid oxidation, improving insulin resistance and decreasing lipogenesis. ${ }^{49,50)}$ Liraglutide, as a GLP-1 analogue, is approved by the U.S. Food and Drug Administration and marketed since 2010 for the treatment of type 2 diabetes due to its glucose-lowering effect. ${ }^{51)}$ Liraglutide has also been reported to reduce hepatic steatosis and endoplasmic reticulum (ER) stress in mice fed with a high fat diet. ${ }^{52)}$ Another GLP-1 analogue, exendin-4, has been demonstrated to decrease the fatty acid load in hepatocytes and reverse hepatic steatosis in obese mice. ${ }^{53,54)}$ In the present study, our results confirmed that liraglutide decreased body weight, increased insulin sensitivity, reduced hepatic lipid accumulation and consequently decreased serum ALT levels, which indicated 
that liraglutide might have a liver protective effect in the rats with HFHC diet-induced NAFLD. Previous studies have reported that MDA levels in the liver were reduced by administration of exendin-4 in ob/ob mice. ${ }^{53)}$ To investigate whether liraglutide also had anti-oxidative and anti-inflammatory properties in the liver, we examined the lipid peroxidation (MDA and FFAs), antioxidant enzyme (SOD) and pro-inflammatory markers in the rats fed with a high fat and high cholesterol diet. Our results demonstrated that these oxidative stress and inflammatory markers were significantly reversed by liraglutide treatment compared to those in the HFHC group. These results indicated that liraglutide can alleviate hepatic oxidative stress and inflammatory response.

Adiponectin is a specific protein secreted by adipocytes, which can regulate glucose and lipid metabolism, and protect the liver against steatosis. ${ }^{54)}$ A large number of studies in experimental animals and patients have found that the level of adiponectin is closely related to obesity, insulin resistance, coronary heart disease and NAFLD. ${ }^{55,56)}$ In addition, adiponectin has been reported to be tightly associated with oxidative stress and inflammatory respons. ${ }^{27)}$ It is plausible that adiponectin is one of the mediators for the anti-oxidative and anti-inflammatory effects of liraglutide. Previous studies have found that two GLP-1 agonists, exendin-4 and Boc5, significantly increase the serum adiponectin levels and consequently reverse hepatic steatosis or insulin resistance in obese mice. ${ }^{53,57)}$ However, whether liraglutide can increase the levels of adiponectin in the serum and liver remain to be elucidated. In the present study, we demonstrated that the level of adiponectin in the serum and liver were significantly increased after liraglutide treatment, which strongly suggests that adiponectin might be involved in the anti-oxidative and anti-inflammatory effects of liraglutide.

Recently, the JNK signal transduction pathway has been shown to play an important role in the pathogenesis and development of NAFLD. ${ }^{29,30)}$ It has been found that the activation of JNKs (mainly JNK1) mediates insulin resistance, steatohepatitis and inflammatory response. ${ }^{31,32)}$ Solinas et al. have reported that knockout of JNK1 in mice alleviates high fat diet-induced inflammatory response and hepatic steatosis and enhances insulin sensitivity. ${ }^{58)}$ In addition, an elevation of serum adiponectin levels has been observed in mice with deletion of JNK1. ${ }^{33)}$ A recent study has demonstrated that liraglutide attenuates the expression of TNF- $\alpha$ and inhibits the activation of JNK1 in the liver. ${ }^{28)}$ Our present results showed that HFHC diet significantly increased the protein expression of JNK1 and p-JNK1 in the liver, and that liraglutide completely reduced the expression of JNK1 and p-JNK1 to the control level. Liraglutide has been shown to repress NF- $\kappa \mathrm{B}$ activation in endothelial cells, ${ }^{59)}$ suggesting that an anti-inflammatory effect may be part of the beneficial function of liraglutide. In our study, liraglutide was found to inhibit TNF- $\alpha$ expression. These results suggest that the inactivation of JNK1 and NF- $\kappa \mathrm{B}$ may contribute to the hepatoprotective effect of liraglutide through the elevation of adiponectin and subsequent suppression of inflammatory response and oxidative stress in the liver.

In conclusion, the present study demonstrated that liraglutide treatment upon high fat and high cholesterol diet in the rats effectively inhibited the oxidative stress and inflammatory response in the liver leading to improvement of insulin resistance, liver function, and hepatic lipid accumulation. The anti- oxidative and anti-inflammatory effects of liraglutide in the liver might be mediated by the elevation of hepatic adiponectin levels and the inactivation of JNK1. Our results contribute to a better understanding of the hepatoprotective effects of liraglutide to further pave the road for the therapeutic potential of this compound in treatment of NAFLD.

Acknowledgments This work was supported by Research Grants from the Natural Science Foundation of Guangdong Province (No. 10151008008000003 and No. S201201001095).

Conflict of Interest The authors declare no conflict of interest.

\section{REFERENCES}

1) Dowman JK, Armstrong MJ, Tomlinson JW, Newsome PN. Current therapeutic strategies in non-alcoholic fatty liver disease. Diabetes Obes. Metab., 13, 692-702 (2011).

2) Fabbrini E, Magkos F, Mohammed BS, Pietka T, Abumrad NA, Patterson BW, Okunade A, Klein S. Intrahepatic fat, not visceral fat, is linked with metabolic complications of obesity. Proc. Natl. Acad. Sci. U.S.A., 106, 15430-15435 (2009).

3) Baffy G. Hepatocellular carcinoma in non-alcoholic fatty liver disease: epidemiology, pathogenesis, and prevention. Journal of Clinical and Translational Hepatology, 1, 131-137 (2013).

4) Schuppan D, Gorrell MD, Klein T, Mark M, Afdhal NH. The challenge of developing novel pharmacological therapies for nonalcoholic steatohepatitis. Liver Int., 30, 795-808 (2010).

5) Farrell GC, Larter CZ. Nonalcoholic fatty liver disease: from steatosis to cirrhosis. Hepatology, 43 (Suppl. 1), S99-S112 (2006).

6) Vanni E, Bugianesi E, Kotronen A, De Minicis S, Yki-Jarvinen H, Svegliati-Baroni G. From the metabolic syndrome to NAFLD or vice versa? Dig. Liver Dis., 42, 320-330 (2010).

7) Kim $\mathrm{CH}$, Younossi ZM. Nonalcoholic fatty liver disease: a manifestation of the metabolic syndrome. Cleve. Clin. J. Med., 75, 721-728 (2008)

8) Day CP, James OF. Steatohepatitis: a tale of two "hits"? Gastroenterology, 114, 842-845 (1998).

9) Bugianesi E, McCullough AJ, Marchesini G. Insulin resistance: a metabolic pathway to chronic liver disease. Hepatology, 42, 9871000 (2005).

10) Chitturi S, Abeygunasekera S, Farrell GC, Holmes-Walker J, Hui JM, Fung C, Karim R, Lin R, Samarasinghe D, Liddle C, Weltman $\mathrm{M}$, George J. NASH and insulin resistance: Insulin hypersecretion and specific association with the insulin resistance syndrome. Hepatology, 35, 373-379 (2002).

11) Mahady SE, George J. Management of nonalcoholic steatohepatitis: an evidence-based approach. Clin. Liver Dis., 16, 631-645 (2012).

12) Kugelmas M, Hill DB, Vivian B, Marsano L, McClain CJ. Cytokines and NASH: a pilot study of the effects of lifestyle modification and vitamin E. Hepatology, 38, 413-419 (2003).

13) Hickman IJ, Jonsson JR, Prins JB, Ash S, Purdie DM, Clouston $\mathrm{AD}$, Powell EE. Modest weight loss and physical activity in overweight patients with chronic liver disease results in sustained improvements in alanine aminotransferase, fasting insulin, and quality of life. Gut, 53, 413-419 (2004).

14) Kim W, Egan JM. The role of incretins in glucose homeostasis and diabetes treatment. Pharmacol. Rev., 60, 470-512 (2008).

15) Lee J, Hong SW, Rhee EJ, Lee WY. GLP-1 receptor agonist and non-alcoholic fatty liver disease. Diabetes Metab. J., 36, 262-267 (2012).

16) Kim YO, Schuppan D. When GLP-1 hits the liver: a novel approach for insulin resistance and NASH. Am. J. Physiol. Gastrointest. Liver Physiol., 302, G759-G761 (2012). 
17) Burmeister MA, Ferre T, Ayala JE, King EM, Holt RM, Ayala JE. Acute activation of central GLP-1 receptors enhances hepatic insulin action and insulin secretion in high-fat-fed, insulin resistant mice. Am. J. Physiol. Endocrinol. Metab., 302, E334-E343 (2012).

18) Lee YS, Park MS, Choung JS, Kim SS, Oh HH, Choi CS, Ha SY, Kang Y, Kim Y, Jun HS. Glucagon-like peptide-1 inhibits adipose tissue macrophage infiltration and inflammation in an obese mouse model of diabetes. Diabetologia, 55, 2456-2468 (2012).

19) Shiraki A, Oyama J, Komoda H, Asaka M, Komatsu A, Sakuma M, Kodama K, Sakamoto Y, Kotooka N, Hirase T, Node K. The glucagon-like peptide 1 analog liraglutide reduces TNF-alpha-induced oxidative stress and inflammation in endothelial cells. Atherosclerosis, 221, 375-382 (2012).

20) Batchuluun B, Inoguchi $T$, Sonoda $N$, Sasaki S, Inoue $T$, Fujimura Y, Miura D, Takayanagi R. Metformin and liraglutide ameliorate high glucose-induced oxidative stress via inhibition of PKC$\mathrm{NAD}(\mathrm{P}) \mathrm{H}$ oxidase pathway in human aortic endothelial cells. Atherosclerosis, 232, 156-164 (2014).

21) Okada K, Kotani K, Yagyu H, Ando A, Osuga JI, Ishibashi S. Effects of treatment with liraglutide on oxidative stress and cardiac natriuretic peptide levels in patients with type 2 diabetes mellitus. Endocrine, 47, 962-964 (2014).

22) Noyan-Ashraf MH, Shikatani EA, Schuiki I, Mukovozov I, Wu J, Li RK, Volchuk A, Robinson LA, Billia F, Drucker DJ, Husain M. A glucagon-like peptide-1 analog reverses the molecular pathology and cardiac dysfunction of a mouse model of obesity. Circulation, 127, 74-85 (2013).

23) Parthsarathy V, Holscher C. The type 2 diabetes drug liraglutide reduces chronic inflammation induced by irradiation in the mouse brain. Eur. J. Pharmacol., 700, 42-50 (2013).

24) Gao H, Xu L, Li D, Guang L, Deng W. Effects of glucagon-like peptide- 1 on liver oxidative stress, TNF- $\alpha$ and TGF- $\beta 1$ in rats with non-alcoholic fatty liver disease. Nan Fang Yi Ke Da Xue Xue Bao, 33, 1661-1664 (2013).

25) Polyzos SA, Kountouras J, Zavos C, Tsiaousi E. The role of adiponectin in the pathogenesis and treatment of non-alcoholic fatty liver disease. Diabetes Obes. Metab., 12, 365-383 (2010).

26) Polyzos SA, Kountouras J, Zavos C. Nonalcoholic fatty liver disease: the pathogenetic roles of insulin resistance and adipocytokines. Curr. Mol. Med., 9, 299-314 (2009).

27) Chen SJ, Yen CH, Huang YC, Lee BJ, Hsia S, Lin PT. Relationships between inflammation, adiponectin, and oxidative stress in metabolic syndrome. PLoS ONE, 7, e45693 (2012).

28) Zhang L, Yang M, Ren H, Hu H, Boden G, Li L, Yang G. GLP-1 analogue prevents NAFLD in ApoE KO mice with diet and Acrp30 knockdown by inhibiting c-JNK. Liver Int., 33, 794-804 (2013).

29) Tan Y, Zhang JN, Chen JH, Wang LJ, Liu HX. Role of JNK signal transduction pathway in nonalcoholic fatty liver disease. Zhonghua Gan Zang Bing Za Zhi, 17, 821-825 (2009).

30) Liu G, Rondinone CM. JNK: bridging the insulin signaling and inflammatory pathway. Curr. Opin. Investig. Drugs, 6, 979-987 (2005).

31) Kodama Y, Kisseleva T, Iwaisako K, Miura K, Taura K, De Minicis S, Osterreicher CH, Schnabl B, Seki E, Brenner DA. c-Jun N-terminal kinase-1 from hematopoietic cells mediates progression from hepatic steatosis to steatohepatitis and fibrosis in mice. Gastroenterology, 137, 1467-1477 e5 (2009).

32) Wu N, Lu Y, He B, Zhang Y, Lin J, Zhao S, Zhang W, Li Y, Han P. Taurine prevents free fatty acid-induced hepatic insulin resistance in association with inhibiting JNK1 activation and improving insulin signaling in vivo. Diabetes Res. Clin. Pract., 90, 288-296 (2010).

33) Hirosumi J, Tuncman G, Chang L, Gorgun CZ, Uysal KT, Maeda $\mathrm{K}$, Karin M, Hotamisligil GS. A central role for JNK in obesity and insulin resistance. Nature, 420, 333-336 (2002).

34) Folch J, Lees M, Sloane Stanley GH. A simple method for the isolation and purification of total lipides from animal tissues. J. Biol.
Chem., 226, 497-509 (1957)

35) Lapenna D, Ciofani G, Pierdomenico SD, Giamberardino MA, Cuccurullo F. Reaction conditions affecting the relationship between thiobarbituric acid reactivity and lipid peroxides in human plasma. Free Radic. Biol. Med., 31, 331-335 (2001).

36) Tinnikov AA, Boonstra R. Colorimetric micro-determination of free fatty acids in plasma using microplate readers. Clin. Chim. Acta, 281, 159-162 (1999).

37) Marklund S, Marklund G. Involvement of the superoxide anion radical in the autoxidation of pyrogallol and a convenient assay for superoxide dismutase. Eur. J. Biochem., 47, 469-474 (1974).

38) Jain SK, Micinski D, Lieblong BJ, Stapleton T. Relationship between hydrogen sulfide levels and HDL-cholesterol, adiponectin, and potassium levels in the blood of healthy subjects. Atherosclerosis, 225, 242-245 (2012).

39) Borovikova LV, Ivanova S, Zhang M, Yang H, Botchkina GI, Watkins LR, Wang H, Abumrad N, Eaton JW, Tracey KJ. Vagus nerve stimulation attenuates the systemic inflammatory response to endotoxin. Nature, 405, 458-462 (2000).

40) Hunt GB, Luff JA, Daniel L, Van den Bergh R. Evaluation of hepatic steatosis in dogs with congenital portosystemic shunts using Oil Red O staining. Vet. Pathol., 50, 1109-1115 (2013).

41) Niki E, Yoshida Y, Saito Y, Noguchi N. Lipid peroxidation: mechanisms, inhibition, and biological effects. Biochem. Biophys. Res. Commun., 338, 668-676 (2005).

42) Festi D, Colecchia A, Sacco T, Bondi M, Roda E, Marchesini G. Hepatic steatosis in obese patients: clinical aspects and prognostic significance. Obes. Rev., 5, 27-42 (2004).

43) Vickers AE. Characterization of hepatic mitochondrial injury induced by fatty acid oxidation inhibitors. Toxicol. Pathol., 37, 78-88 (2009).

44) Nieto-Vazquez I, Fernandez-Veledo S, Kramer DK, Vila-Bedmar R, Garcia-Guerra L, Lorenzo M. Insulin resistance associated to obesity: the link TNF-alpha. Arch. Physiol. Biochem., 114, 183-194 (2008).

45) Borst SE. The role of TNF-alpha in insulin resistance. Endocrine, 23, 177-182 (2004).

46) Zelko IN, Mariani TJ, Folz RJ. Superoxide dismutase multigene family: a comparison of the CuZn-SOD (SOD1), Mn-SOD (SOD2), and EC-SOD (SOD3) gene structures, evolution, and expression. Free Radic. Biol. Med., 33, 337-349 (2002).

47) Yang H, Liu C, Yang D, Zhang H, Xi Z. Comparative study of cytotoxicity, oxidative stress and genotoxicity induced by four typical nanomaterials: the role of particle size, shape and composition. $J$. Appl. Toxicol., 29, 69-78 (2009).

48) Jung JH, Kim HS. The inhibitory effect of black soybean on hepatic cholesterol accumulation in high cholesterol and high fat dietinduced non-alcoholic fatty liver disease. Food Chem. Toxicol., 60, 404-412 (2013)

49) Monji A, Mitsui T, Bando YK, Aoyama M, Shigeta T, Murohara T. Glucagon-like peptide-1 receptor activation reverses cardiac remodeling via normalizing cardiac steatosis and oxidative stress in type 2 diabetes. Am. J. Physiol. Heart Circ. Physiol., 305, H295-H304 (2013).

50) Svegliati-Baroni G, Saccomanno S, Rychlicki C, Agostinelli L, De Minicis S, Candelaresi C, Faraci G, Pacetti D, Vivarelli M, Nicolini D, Garelli P, Casini A, Manco M, Mingrone G, Risaliti A, Frega GN, Benedetti A, Gastaldelli A. Glucagon-like peptide-1 receptor activation stimulates hepatic lipid oxidation and restores hepatic signalling alteration induced by a high-fat diet in nonalcoholic steatohepatitis. Liver Int., 31, 1285-1297 (2011).

51) Parks M, Rosebraugh C. Weighing risks and benefits of liraglutidethe FDA's review of a new antidiabetic therapy. N. Engl. J. Med., 362, 774-777 (2010).

52) Sharma S, Mells JE, Fu PP, Saxena NK, Anania FA. GLP-1 analogs reduce hepatocyte steatosis and improve survival by enhancing the 
unfolded protein response and promoting macroautophagy. PLoS ONE, 6, e25269 (2011).

53) Ding X, Saxena NK, Lin S, Gupta NA, Anania FA. Exendin-4, a glucagon-like protein-1 (GLP-1) receptor agonist, reverses hepatic steatosis in ob/ob mice. Hepatology, 43, 173-181 (2006).

54) Méndez-Sánchez N, Chávez-Tapia NC, Villa AR, Sánchez-Lara $\mathrm{K}$, Zamora-Valdés D, Ramos MH, Uribe M. Adiponectin as a protective factor in hepatic steatosis. World J. Gastroenterol., 11, 1737-1741 (2005).

55) Polyzos SA, Toulis KA, Goulis DG, Zavos C, Kountouras J. Serum total adiponectin in nonalcoholic fatty liver disease: a systematic review and meta-analysis. Metabolism, 60, 313-326 (2011).

56) Sheng T, Yang K. Adiponectin and its association with insulin resistance and type 2 diabetes. J. Genet. Genomics, 35, 321-326 (2008).
57) He M, Su H, Gao W, Johansson SM, Liu Q, Wu X, Liao J, Young AA, Bartfai T, Wang MW. Reversal of obesity and insulin resistance by a non-peptidic glucagon-like peptide-1 receptor agonist in diet-induced obese mice. PLoS ONE, 5, e14205 (2010).

58) Solinas G, Vilcu C, Neels JG, Bandyopadhyay GK, Luo JL, Naugler W, Grivennikov S, Wynshaw-Boris A, Scadeng M, Olefsky JM, Karin M. JNK1 in hematopoietically derived cells contributes to diet-induced inflammation and insulin resistance without affecting obesity. Cell Metab., 6, 386-397 (2007).

59) Dai Y, Mehta JL, Chen M. Glucagon-like peptide-1 receptor agonist liraglutide inhibits endothelin-1 in endothelial cell by repressing nuclear factor-kappa B activation. Cardiovasc. Drugs Ther., 27, 371-380 (2013). 